\title{
Comparison of the chemical constituents and anti-Alzheimer's disease effects of Uncaria rhynchophylla and Uncaria tomentosa
}

Qing-Qing Xu', Pang Chui Shaw ${ }^{2,3}$, Zhen Hu' ${ }^{1}$, Wen Yang ${ }^{1}$, Siu-Po Ip ${ }^{1}$, Yan-Fang Xian ${ }^{1 *}$ (D) and Zhi-Xiu Lin ${ }^{1,3,4^{*}}$

\begin{abstract}
Background: Uncaria tomentosa, which has similar chemical constituents with Uncaria rhynchophylla, has been reported to alleviate cognitive impairments in Alzheimer's disease (AD) animal models. This study aimed to compare the chemical constituents and anti-AD effect of the ethanol extracts of U. tomentosa (UTE) and U. rhynchophylla (URE).

Methods: The high-performance liquid chromatography (HPLC) was used to compare the chemical constituents of UTE and URE. Streptozotocin (STZ) was intracerebroventricularly (ICV) injected into adult male Sprague-Dawley (SD) rats to establish AD model. UTE (400 mg/kg) or URE (400 mg/kg) was administrated intragastrically once daily to the rats for 6 consecutive weeks. Morris water maze (MWM) test was conducted to assess the neurological functions in the STZ-induced AD rats. The brain tissues of the rats were harvested for further biochemical assay.

Results: The MWM test results showed both UTE and URE could significantly improve the learning and memory impairments induced by STZ in rats. Both UTE and URE could significantly inhibit the hyperphosphorylation of tau protein, reduce the elevated levels of pro-inflammatory cytokines (IL-1 $\beta$, IL- 6 and TNF-a), enhance activities of antioxidant enzymes (SOD, CAT and GPX) and increase the protein expression of HO-1. In addition, UTE could decrease the malondialdehyde (MDA) level. Furthermore, both UTE and URE significantly enhanced Akt activation, down regulated the activation of glycogen synthase kinase $3 \beta$ (GSK-3 $\beta$ ), and induced the nuclear translocation of Nrf2 in the STZinduced $A D$ rats.

Conclusions: UTE and URE contained similar chemical constituents. We found for the first time that both of them could ameliorate cognitive deficits in the STZ-induced AD rats. The underlying molecular mechanism involve suppression of tau hyperphosphorylation, anti-oxidant and anti-neuroinflammation via modulating Akt (Ser473)/GSK3 $\beta$ (Ser9)-mediated Nrf2 activation. These findings amply implicate that both of UTE and URE are worthy of being developed clinically into pharmaceutical treatment for AD.
\end{abstract}

Keywords: Uncaria rhynchophylla, Uncaria tomentosa, Alzheimer's disease, Streptozotocin, Rats, Akt/GSK3ß/Nrf2 pathway

*Correspondence: lisaxian@cuhk.edu.hk; linzx@cuhk.edu.hk ${ }^{1}$ School of Chinese Medicine, Faculty of Medicine, The Chinese University of Hong Kong, Shatin, N.T., Hong Kong SAR, People's Republic of China Full list of author information is available at the end of the article

\begin{abstract}
Background
Alzheimer's disease (AD) is a chronic neurodegenerative disease characterized by impaired memory and cognitive functions, and seriously affects one's daily living. AD is the most common cause of dementia, accounting for $60-70 \%$ of all cases of dementia [1]. Approximately 9.8 million people in China and 47 million people worldwide are currently affected by $\mathrm{AD}$ or related dementia, and
\end{abstract} original author(s) and the source, provide a link to the Creative Commons licence, and indicate if changes were made. The images or other third party material in this article are included in the article's Creative Commons licence, unless indicated otherwise in a credit line to the material. If material is not included in the article's Creative Commons licence and your intended use is not permitted by statutory regulation or exceeds the permitted use, you will need to obtain permission directly from the copyright holder. To view a copy of this licence, visit http://creativecommons.org/licenses/by/4.0/. The Creative Commons Public Domain Dedication waiver (http://creativeco mmons.org/publicdomain/zero/1.0/) applies to the data made available in this article, unless otherwise stated in a credit line to the data. 
this figure is estimated to triple by 2050 [1, 2]. Moreover, according to a recent report released by World Health Organization, AD became the second biggest killer in high-income countries as well as the seventh leading cause of death worldwide in 2019 [3]. Clearly, AD poses a huge threat to public health, and brings a heavy burden to the patients' families and societies as a whole.

There is no cure or disease-modifying treatment for $\mathrm{AD}$ at present, partly owing to the complex pathogenesis of the disease. Despite its multifactorial etiopathogenesis, two pathological hallmarks in $\mathrm{AD}$ brain are believed to contribute to the progression of the disease [4], i.e., neurofibrillary tangles (NFTs) principally consisting of hyperphosphorylated tau protein, and extracellular amyloid plaques mainly composed of aggregated beta-amyloid $(A \beta)$ peptide. Other pathological features, such as neuroinflammation, oxidative stress, synaptic loss and neuronal degeneration, have also been recognized to contribute to the progression of the disease [5]. Mounting evidence suggests that oxidative stress and neuroinflammation play cardinal roles in the early stage of $\mathrm{AD}$ as they are interconnected and increase the deposition of both $A \beta$ and tau [6]. However, the consecutive failures of anti-A $\beta$ therapeutics in clinical trials recently indicate that neither $A \beta$ clearance nor other pathological features should be taken as individual targets $[6,7]$. The therapeutics now commonly used in clinic are namely acetylcholinesterase inhibitors (AChEIs; e.g., donepezil, rivastigmine and galantamine) and non-competitive $N$-methyl-D-aspartate (NMDA) receptor antagonist (e.g., memantine), but they can only partially relieve the symptoms for a limited period of time [8]. Thus far, these drugs only provide temporary symptomatic relief for $\mathrm{AD}$ patients, but cannot delay or stop the underlying pathology [9]. In addition, due to the occurrence of drug resistance and undesirable side effects, these anti-AD drugs have limitations in clinical use [10]. Given the apparent unmet medical need for AD treatment, there clearly exists a need to seek alternative therapeutic approaches.

The etiology of $\mathrm{AD}$ is known to be multifactorial, while many traditional medicines and natural products are multi-components and can act on multiple molecular targets to afford the neuroprotective effect in the management of $\mathrm{AD}[11,12]$. Historically, Chinese herbal medicine (CHM) has been used to treat neurodegenerative disorders and is still widely prescribed in many Eastern Asian countries nowadays [13]. Uncariae Ramulus Cum Uncis (also known as Gou-teng in Chinese) is derived from the stem with hook of Uncaria rhynchophylla (Uncaria). This herb is one of the most commonly prescribed herbs in Asia, and has been extensively used in Chinese medicine practice to treat a variety of cerebral diseases, such as epilepsy, convulsion, headache and hypertension [14]. It is also incorporated into a number of herbal formulae which are prescribed for the treatment of AD, such as Chotosan (Gouteng-San in Chinese) and Yokukansan (Yigan-San in Chinese) $[15,16]$. Our previous study has demonstrated that $U$. rhynchophylla and its major components have potent neuroprotective effects on neurodegenerative diseases, such as AD. Specifically, $U$. rhynchophylla was found to ameliorate cognitive deficits in a mouse model of $\mathrm{AD}$, and the mechanism was related to the inhibition of AChE activity and the enhancement of antioxidant activity [17]. Furthermore, previous studies in our laboratory also revealed that isorhynchophylline (IRN), the major chemical ingredient of $U$. rhynchophylla, played a predominant role in neuroprotection and exerted extensive bioactivities in both in vitro and in vivo models of AD [18-22].

Cat's claw, whose name comes from the shape of the crooked thorns on its woody vines, is indigenous to the Amazon River basin [23]. It is one of the best-selling herbal remedies in the United States and European countries [23]. Its popularity in the alternative medicine market may be attributed to its purported abilities to strengthen the immune system, inhibit inflammation, inhibit tumor growth, and suppress viral replication [24-26]. Uncaria tomentosa (U. tomentosa), one of the most common species of cat's claw with higher alkaloid content than other species of cat's claw, is preferred as raw material for Cat's claw [23]. U. tomentosa has been known to possess extensive pharmacological activities, including anti-oxidative, anti-inflammatory, immune modulatory and anti-tumor activities [27]. Recent studies showed that $U$. tomentosa could alleviate the cognitive and memory impairments in both APP transgenic mice and middle-aged rats $[28,29]$. However, the potency of the anti-AD effect of $U$. rhynchophylla and $U$. tomentosa has not been compared so far. Moreover, the molecular mechanisms underlying the neuroprotective effects of $U$. tomentosa still remains unexplored. $U$. rhynchophylla is used interchangeably with other Uncaria species, such as $U$. tomentosa, in clinical application, because of the similarity in chemical constituents. Thus, in the present study, we aimed to compare the chemical constituents and antiAD activity of $U$. rhynchophylla and $U$. tomentosa.

\section{Methods}

\section{Chemicals and reagents}

Donepezil hydrochloride was purchased from SigmaAldrich (St. Louis, MO, USA). Streptozotocin (STZ, purity $\geq 98 \%$ ) was purchased from Santa Cruz Biotechnology (Dallas, USA). Ethanol was obtained from DAEJUNG Chemicals (Gyeonggi-do, Korea). Methanol (HPLC grade) was bought from Duksan Pure Chemicals (Gyeonggi-do, Korea). Triethylamine (HPLC grade) 
was purchased from Scharlau (Barcelona, Spain). Standard substances (rhynchophylline, isorhynchophylline, corynoxeine and isocorynoxeine, purity $\geq 98 \%$ ) were purchased from Chengdu Mansite Pharmacetical Co. Ltd (Chengdu, China). All other chemicals and reagents used were of analytical grade.

\section{Extraction and quality control of herbal materials}

The Uncariae Ramulus Cum Uncis originated from the dried stem with hooks of Uncaria rhynchophylla was purchased from Zhixin Pharmaceutical Co., Ltd, a GMPaccredited Chinese herbal supplier based in Guangzhou, Guangdong Province, China. Uncaria tomentosa was purchased from Hking Bio-Tech Co., Ltd (Changsha, Hunan Province, China). They were authenticated to be the dried stem with hooks of Uncaria rhynchophylla (Miq.) Jacks. and Uncaria tomentosa, respectively, by Ms. Y.Y. Zong, a seasoned pharmacognist at the School of Chinese Medicine, The Chinese University of Hong Kong, where voucher specimens (No.091220 and 091221, respectively) were deposited. For each raw herb, the dried herb was ground to powder or pieces. The herb $(1000 \mathrm{~g})$ was macerated in $10 \mathrm{~L}$ of $70 \%$ aqueous ethanol for $24 \mathrm{~h}$ at room temperature, and then extracted in an ultrasonic bath for $1 \mathrm{~h}$. Extractions were repeated twice. Following filtration, the crude extracts were centrifuged to remove undissolved particles. The pooled extracts were combined and concentrated under reduced pressure, followed by freeze drying. The yields of the extracts of $U$. tomentosa (UTE) and $U$. rhynchophylla (URE) were $14.62 \%$ and $13.88 \%$, respectively. The dried aqueous extracts were stored in desiccators at room temperature and avoid sunlight.

The high-performance liquid chromatography (HPLC) profiles of UTE and URE were constructed using an ACQUITY UPLC system (Waters, USA) equipped with a PDA e $\lambda$ detector, a FTN sample manager, and quaternary solvent manager. Briefly, UTE or URE was dissolved in methanol and injected into UPLC by an autosampler. A Nucleosil 100 C18 HPLC column $(4.6 \mathrm{~mm} \times 250 \mathrm{~mm}$, $5 \mu \mathrm{m})$ was used for separation. The mobile phase consisted of $0.01 \mathrm{mmol} / \mathrm{L}$ triethylamine in water (solvent A) and methanol (solvent B). Separation was achieved by a linear gradient elution from 60 to $85 \%$ solvent B over $40 \mathrm{~min}$ at a flow rate of $1.0 \mathrm{~mL} / \mathrm{min}$. The separation temperature was set at room temperature $\left(25^{\circ} \mathrm{C}\right)$ and detection wavelength was at $245 \mathrm{~nm}$. The sample injection volume was $10 \mu \mathrm{L}$.

Method validation was performed based on a published procedure [30]. Calibration curves were established by plotting the peak area versus concentration of each analyte using a least-squares linear regression analysis. Limits of detection (LOD) and limits of quantification
(LOQ) for each analyte were defined as signal-to-noise ratios $(\mathrm{S} / \mathrm{N})$ of 3 and 10 , respectively. The precisions were determined by analyzing the five replicates of reference substances on the same day. Recovery tests were performed by spiking the raw material with reference standards at three levels, and then subjecting the solutions to the same sample preparation. Three replicates were performed for each analysis. To confirm the repeatability, five replicates of the same sample (UTE or URE) were analyzed. Variations were expressed in terms of relative standard deviation (RSD) in all of the tests.

\section{Animals}

Adult male Sprague-Dawley (SD) rats weighing 230$250 \mathrm{~g}$ were obtained from the Laboratory Animal Services Centre, The Chinese University of Hong KONG. Rats were housed in controlled conditions (5 rats per cage, $12 \mathrm{~h} \mathrm{light/dark}$ cycle with a constant room temperature of $20-24{ }^{\circ} \mathrm{C}$ and relative humidity of $40-60 \%$ ) and had free access to food and water. This study was conducted in accordance with the Guide for the Care and Use of Laboratory Animals issued by the National Institutes of Health (NIH Publication No. 85-23, revised 2011). Approval for the experimental protocol was obtained from the Animal Experimentation Ethics Committee of The Chinese University of Hong Kong (Ref. No.18/264/MIS). The utmost possible efforts were made to minimize the number of animals used for this study and the experiment-induced suffering of the experimental animals.

\section{STZ-induced AD model}

AD model was induced by intracerebroventricular (ICV) injection of STZ as previously described [14] with minor modifications. Briefly, rats were fixed on the stereotaxic apparatus (Stoelting, Wood Dale, IL, USA) under anesthesia with an intraperitoneal injection of ketamine $(75 \mathrm{mg} / \mathrm{kg})$ and xylazine $(10 \mathrm{mg} / \mathrm{kg})$. After a midline sagittal incision was made in the scalp, small burr holes ( $1 \mathrm{~mm}$ in diameter) were drilled. STZ $(2 \mathrm{mg} / \mathrm{kg}$, in a volume of $2 \mu \mathrm{L} /$ ventricle, dissolved in $0.05 \mathrm{M}$ citrate buffer, $\mathrm{pH}$ 4.5) was bilaterally injected into the lateral ventricle at a rate of $1 \mu \mathrm{L} / \mathrm{min}$. The stereotaxic coordinates for ICV injection were as follows: $0.8 \mathrm{~mm}$ posterior to bregma, $\pm 1.4 \mathrm{~mm}$ lateral to sagittal suture and $4.0 \mathrm{~mm}$ ventral from the surface of the skull [31]. The sham-operated rats were subjected to the same surgical procedures but injected with the same volume of vehicle $(0.05 \mathrm{M}$ citrate buffer, $\mathrm{pH} 4.5$ ). Rats had free access to food and water after recovery from anesthesia. Each rat received ICV injection of STZ or vehicle twice (on day 1 and day 3 , respectively). 


\section{Animal grouping and drug administration}

Rats were randomly divided into five groups $(n=10)$ : Sham group, STZ+vehicle (STZ-treated control) group, STZ+UTE (400 $\mathrm{mg} / \mathrm{kg})$ group, STZ+URE (400 $\mathrm{mg} / \mathrm{kg}$ ) group, and STZ+Donepezil $(5 \mathrm{mg} / \mathrm{kg}$ ) group. Donepezil was used as the positive control as described previously [20-22]. Drug treatment was initiated at one day after the second STZ injection. UTE, URE and donepezil hydrochloride were suspended in $\mathrm{ddH}_{2} \mathrm{O}$, and administered intragastrically once daily for 6 consecutive weeks, while the same volume of the $\mathrm{ddH}_{2} \mathrm{O}$ was given to the rats in the Sham group and the STZ-treated group for the same duration.

\section{Morris water maze (MWM) test}

The experimental apparatus consisted of a circular pool $(150 \mathrm{~cm}$ in diameter, $45 \mathrm{~cm}$ in height), filled to a depth of $30 \mathrm{~cm}$ with water at $24{ }^{\circ} \mathrm{C}$. The pool was conceptually divided into four equal quadrants for descriptive data collection. A circular escape platform $(10 \mathrm{~cm}$ in diameter) was submerged $1 \mathrm{~cm}$ below the water surface in the midpoint of one quadrant (target quadrant). Some different geometric cues were equipped surrounding the pool, which can be used by experimental animals to determine the platform's location. Rats were trained to find the hidden platform with 3 trials per day for four consecutive days. Each rat was placed in the water facing the pool wall from different release positions. The escape latency to climb onto the platform of each trail was recorded. The maximum trial time was $60 \mathrm{~s}$. If a rat failed to find the platform within $60 \mathrm{~s}$, it was manually guided to the platform and allowed to stay on it for $10 \mathrm{~s}$ (except for $30 \mathrm{~s}$ on the first day), and the escape latency was recorded as $60 \mathrm{~s}$. The probe test was conducted without the hidden platform $24 \mathrm{~h}$ after the last training day. Rats were allowed to swim freely in the water for $60 \mathrm{~s}$. A computerized video tracking system (SuperMaze V2.0 software, Shanghai Xinruan Information Technology Co., Ltd., China) was used to record the swimming paths, the numbers of the target quadrant crossing, the time spent in the target quadrant, and the swimming speed.

\section{Preparation of brain tissue samples}

Twenty-four hours after the MWM test, rats were sacrificed under deep anesthesia. Then the rats were transcardially perfused with ice-cold saline $(0.9 \%)$ until the fluid exiting the right atrium was entirely clear to remove peripheral blood from the CNS vasculature. The hippocampal tissues were rapidly isolated from the brains on ice and were stored at $-80^{\circ} \mathrm{C}$ until use.

\section{Measurement of cytokines}

Hippocampal tissues were homogenized in lysis buffer (Abcam) to prepare $10 \%(\mathrm{w} / \mathrm{v})$ homogenates. The homogenates were centrifuged at $10,000 \mathrm{~g}$ for $15 \mathrm{~min}$ at $4{ }^{\circ} \mathrm{C}$. The supernatants were collected for assay and then for protein determination using a BCA Protein Assay kit (Invitrogen, USA). The levels of interleukin-1 $\beta$ (IL-1 $\beta$ ), IL- 6 and tumor necrosis factor alpha (TNF- $\alpha$ ) in the hippocampal tissues were measured using commercially available enzyme-linked immunosorbent assay (ELISA) kits (IL- $1 \beta$ and TNF- $\alpha$ from Abcam; IL- 6 from RayBiotech) according to the manufacturer's instructions.

\section{Measurement of the levels of superoxide dismutases (SOD), catalase (CAT), malondialdehyde (MDA) and glutathione peroxidase (GPX) activities}

To evaluate the effects of UTE and URE on oxidative stress in the STZ-induced AD model, the activity levels of SOD, CAT, MDA and GPx were assessed. For these biochemical analyses, the brain tissues were homogenized and measured using SOD, CAT, MDA and GPx assay kits (Cat. No. 706002, 707002, 10009055 and 703102, respectively, Cayman, USA) following the manufacturer's protocols. All samples were measured in duplicate.

\section{Western blot analysis}

Approximately $20 \mathrm{mg}$ of the hippocampus tissues were lysed in pre-chilled radio-immune precipitation assay buffer (RIPA; Invitrogen, USA) with a protease inhibitor cocktail (Sigma-Aldrich, USA) to prepare $10 \%(\mathrm{w} / \mathrm{v})$ brain homogenates. The homogenates were centrifuged at $13,000 \mathrm{rmp}$ for $20 \mathrm{~min}$ at $4{ }^{\circ} \mathrm{C}$, and the supernatants were collected. The concentrations of proteins were determined using BCA Protein Assay kit (Invitrogen, USA). Equal amounts $(30 \mu \mathrm{g})$ of denatured protein samples were separated on $10 \%$ SDS-polyacrylamide gels (SDS-PAGE), and transferred to polyvinyl difluoride (PVDF) membranes. After being blocked with 5\% non-fat dry milk for $2 \mathrm{~h}$, the membranes were rinsed and incubated overnight at $4{ }^{\circ} \mathrm{C}$ with primary antibodies, including mouse anti-Tau (Tau 46) antibody (1:500, Santa Cruz, sc-32274), mouse anti-Tau (Tau 5) antibody (1:500, Santa Cruz, sc-58860), rabbit anti-p-Tau (Thr181) antibody (1:1000, Cell Signaling, 12885S), rabbit anti-p-Tau (Ser396) antibody (1:10,000, Abcam, ab109390), rabbit anti-p-Tau (Ser404) antibody (1:1000, Abcam, ab92676), rabbit anti-Keap1 antibody (1:500, Santa Cruz), rabbit anti-Nrf2 antibody (1:1000, Abcam, ab31163), mouse anti-HO1 antibody (1:1000, Abcam, ab13248), rabbit anti-Akt antibody (1:1000, Cell Signaling, 4691P), rabbit anti-p-Akt (Ser473) antibody (1:1000, Cell Signaling, 4060S), rabbit anti-GSK-3 $\beta$ antibody (1:500, Santa Cruz, sc-9166), rabbit anti-p-GSK-3 $\beta$ (Ser9) antibody (1:1000, 


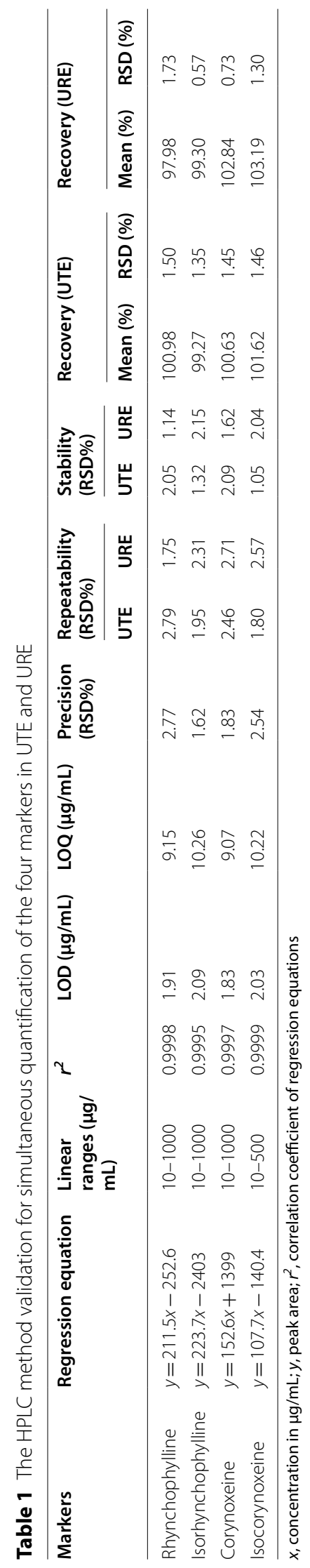


Cell Signaling, 9366S), rabbit Histone H3 antibody (1:500, Santa Cruz, sc-10809) and rabbit anti-GAPDH antibody (1:5000, Abcam, ab181602). Then, the membranes were treated with anti-rabbit IgG secondary antibody (1:3000, Cell Signaling, 7074S) or anti-mouse IgG secondary antibody (1:3000, Cell Signaling, 7076S) for $2 \mathrm{~h}$ at room temperature. The protein bands were visualized using enhanced chemiluminescence (ECL; Invitrogen, WP20005) reagent. Densitometric analysis was performed using Image J software.

\section{Statistical analysis}

All data were presented as means \pm standard deviation $($ mean \pm SD) and all statistical analyses were performed using SPSS 20.0 software (SPSS Inc, Chicago, IL, USA). Group differences in the escape latency in the MWM training task were analyzed using two-way analysis of variance (ANOVA) with repeated measures, with the factors being treatment and training day. The other differences between multiple groups were analyzed by Oneway ANOVA and differences between two groups were analyzed using t-test. A difference was considered statistically significant when $p<0.05$.

\section{Results}

\section{HPLC profile of UTE and URE}

Four representative compounds such as corynoxeine, isocorynoxeine and rhynchophylline and isorhynchophylline were used as chemical markers for the quality control of UTE and URE. Good linear relationships for all compounds were demonstrated $\left(r^{2} \geq 0.9995\right)$. Other HPLC method validation results including linear ranges, LOD, LOQ, precisions, repeatabilities, stabilities, and recoveries were shown in Table 1 . Based on the calculation on the calibration curves of standard substances, the contents of four representative compounds in UTE and URE were shown as follows: UTE contains $0.278 \%$ rhynchophylline, $0.531 \%$ isorhynchophylline, $0.010 \%$ corynoxeine, and $0.028 \%$ isocorynoxeine, respectively; on the other hand, URE contains $0.478 \%$ rhynchophylline, $0.163 \%$ isorhynchophylline, $0.178 \%$ corynoxeine, and $0.418 \%$ isocorynoxeine, respectively. The HPLC chromatogram profiles of UTE and URE were shown in Fig. 1.

\section{Effects of UTE and URE on the cognitive impairments in the STZ-induced AD rats}

MWM test was used to determine learning and spatial memory in the STZ-induced AD rat model. The rats in the STZ+vehicle group had a longer escape latency to find the hidden platform than the Sham group during the training days (day 2, $p<0.05$; day $3, p<0.01$ ), indicating that STZ treatment caused cognitive impairment to the rats. Compared with the STZ+ vehicle group, the rats in both STZ+ UTE group and STZ + URE group took a shorter time to find the platform but without statistically significant difference during the training days (from day 2 to day 4). In the probe trial, when compared with the Sham group, there was conspicuous reductions in the number of crossing target quadrant and time spent in the target quadrant in the STZ-treated group $(p<0.05$ and $p<0.01$, respectively), which was significantly reversed by the treatment of Donepezil. The STZ treated rats with the administration of both UTE and URE markedly spent more time in the target quadrant than vehicle treatment $(p<0.01$ and $p<0.05$, respectively). No statistical differences were observed in the swimming speed among all groups. These experimental findings indicated that both UTE and URE could ameliorate cognitive impairments in the STZ-induced AD rats (Fig. 2).

\section{Effects of UTE and URE on Tau pathology in the STZ-induced AD rats}

The protein expressions of total Tau (Tau-46 and Tau-5) in the STZ+ vehicle group were significantly increased when compared with the Sham group $(p<0.01)$. Both of the STZ+UTE group and STZ+ URE group had statistically lower protein expressions of total Tau than the $\mathrm{STZ}+$ vehicle group ( $p<0.05$ for both). Donepezil treatment $(5 \mathrm{mg} / \mathrm{kg})$ could suppress the level of Tau- 46 when compared with the STZ-treated control group. Moreover, the protein levels of p-Tau (T181), p-Tau (S396) and p-Tau (S404) in the STZ control group were remarkably elevated $(p<0.01)$ when compared with the Sham group. UTE treatment could inhibit the hyperphosphorylation of Tau protein at the site of S396 $(p<0.05)$ and S404 $(p<0.05)$ when compared with the STZ control group, while URE treatment could reverse the protein expressions of $\mathrm{p}$-Tau $(\mathrm{T} 181)(p<0.05)$ and $\mathrm{p}$-Tau $(\mathrm{S} 396)$ $(p<0.01)$. Similarly, Donepezil treatment $(5 \mathrm{mg} / \mathrm{kg})$ efficiently reduced the protein expression of p-Tau (S396) $(p<0.01)$, when compared with the STZ control group (Fig. 3).

\section{Effects of UTE and URE on the expression levels of pro-inflammatory cytokines in the STZ-induced AD rats} Compared with the Sham group, there was a significantly enhanced levels of IL-1 $\beta$, IL- 6 and TNF- $\alpha$ in the STZ+ vehicle group $(p<0.01)$. Treatment with UTE or Donepezil efficiently suppressed the elevated levels of IL-1 $\beta$ ( $p<0.05$ for both), IL-6 $(p<0.01$ and $p<0.05$, respectively) and TNF- $\alpha(p<0.01$ and $p<0.05$, respectively) when compared with the STZ control group. URE treatment markedly inhibited the level of IL-6 $(p<0.05)$ and decreased the levels of IL- $1 \beta$ and TNF- $\alpha$ but without statistically significant difference, as compared with the STZ control group (Fig. 4). 


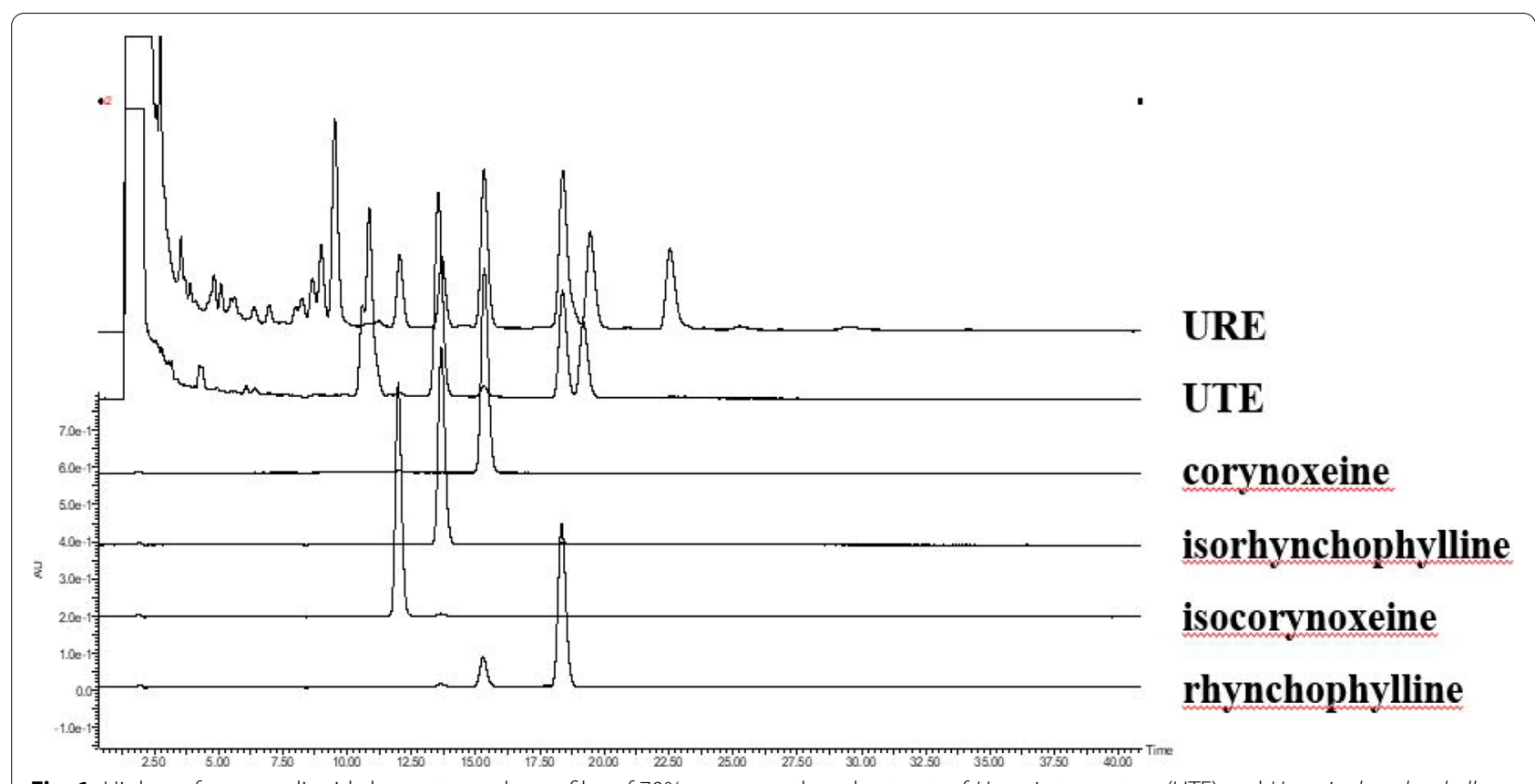

Fig. 1 High-performance liquid chromatography profiles of 70\% aqueous ethanol extracts of Uncaria tomentosa (UTE) and Uncaria rhynchophylla (URE). 1: isorhynchophylline; 2: corynoxeine; 3: rhynchophylline; 4: isocorynoxeine

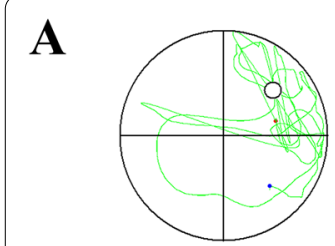

Sham

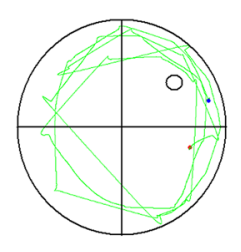

STZ

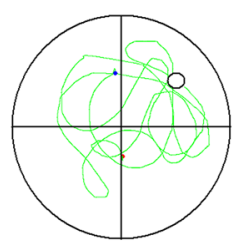

STZ+UTE

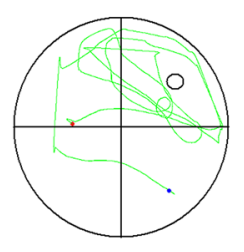

STZ+URE

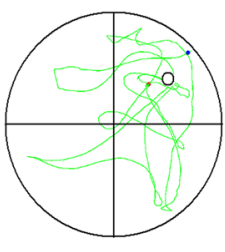

STZ+Donepezil
B

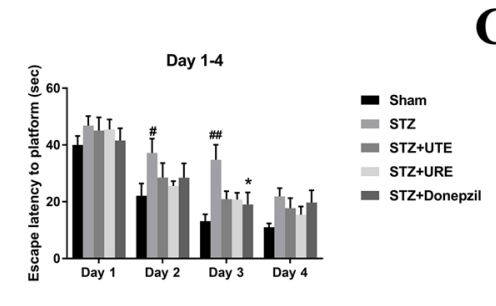

C

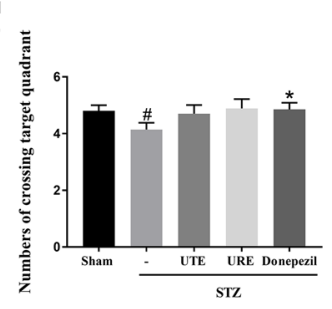

D

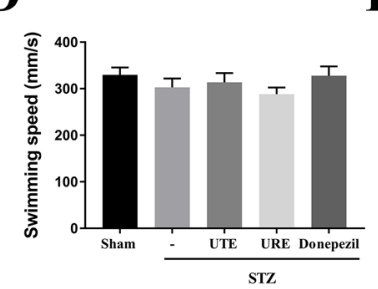

$\mathbf{E}$

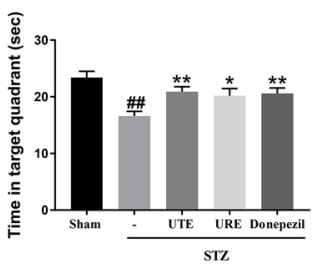

Fig. 2 Effects of UTE and URE on the cognitive impairments in the STZ-induced AD rat model assessed by Morris Water Maze test $(n=9-10)$. A Representative images of the swimming path; $\mathbf{B}$ escape latency to platform during training days; $\mathbf{C}$ the number of target quadrant crossing in the probe tests; $\mathbf{D}$ swimming speed; $\mathbf{E}$ the time spent in the target quadrant in the probe test. Data were expressed as the mean \pm SEM. ${ }^{\#} p<0.05$ and ${ }^{\# \#} p<0.01$ compared with the sham control group. ${ }^{*} p<0.05$ and ${ }^{* *} p<0.01$ compared with the STZ control group 


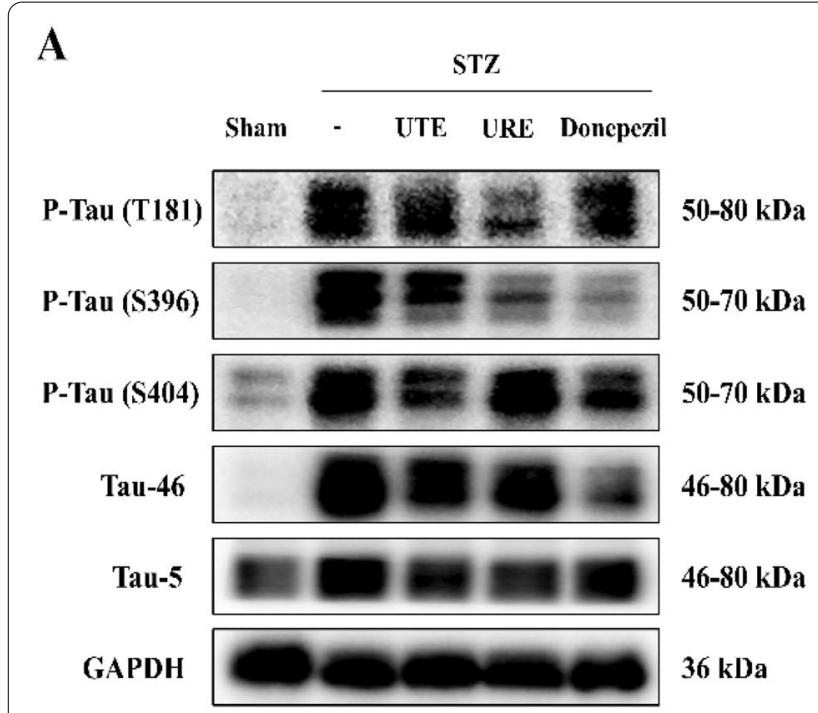

B
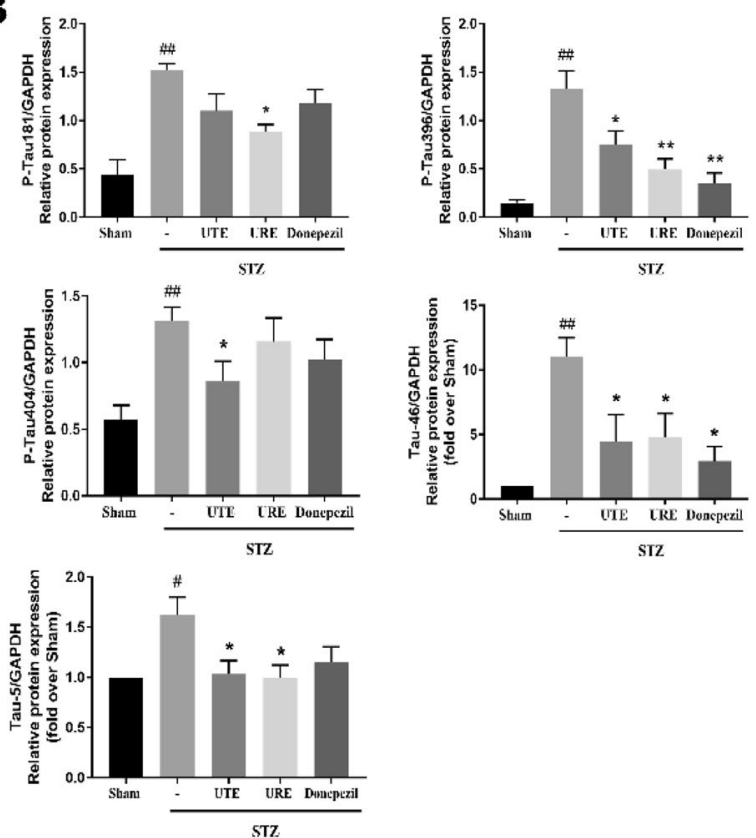

Fig. 3 Effects of UTE and URE on tau hyperphosphorylation on the STZ-induced AD rat model $(n=5)$. Data were expressed as the mean \pm SEM. ${ }^{\#} p<0.05$ and ${ }^{\# \#} p<0.01$ compared with the sham control group. ${ }^{*} p<0.05$ and ${ }^{* *} p<0.01$ compared with the STZ control group

\section{Effects of UTE and URE on oxidative stress} in the STZ-induced $A D$ rats

To assess the antioxidant potential of UTE and URE in the STZ-induced AD rats, the levels of oxidative damage marker (MDA), the antioxidant enzyme activities (SOD, CAT and GPx) and the protein expression of HO-1were measured. The SOD activity significantly decreased in the $\mathrm{STZ}+$ vehicle group $(p<0.01)$ as compared with the Sham group. Both URE and Donepezil treatment markedly $(p<0.01$ and $p<0.05$, respectively) increased the SOD activities as compared with the STZ+ vehicle group. The STZ+UTE group had a higher level of SOD activity than the STZ+ vehicle group, but no significant difference was found between the two groups (Fig. 5A).
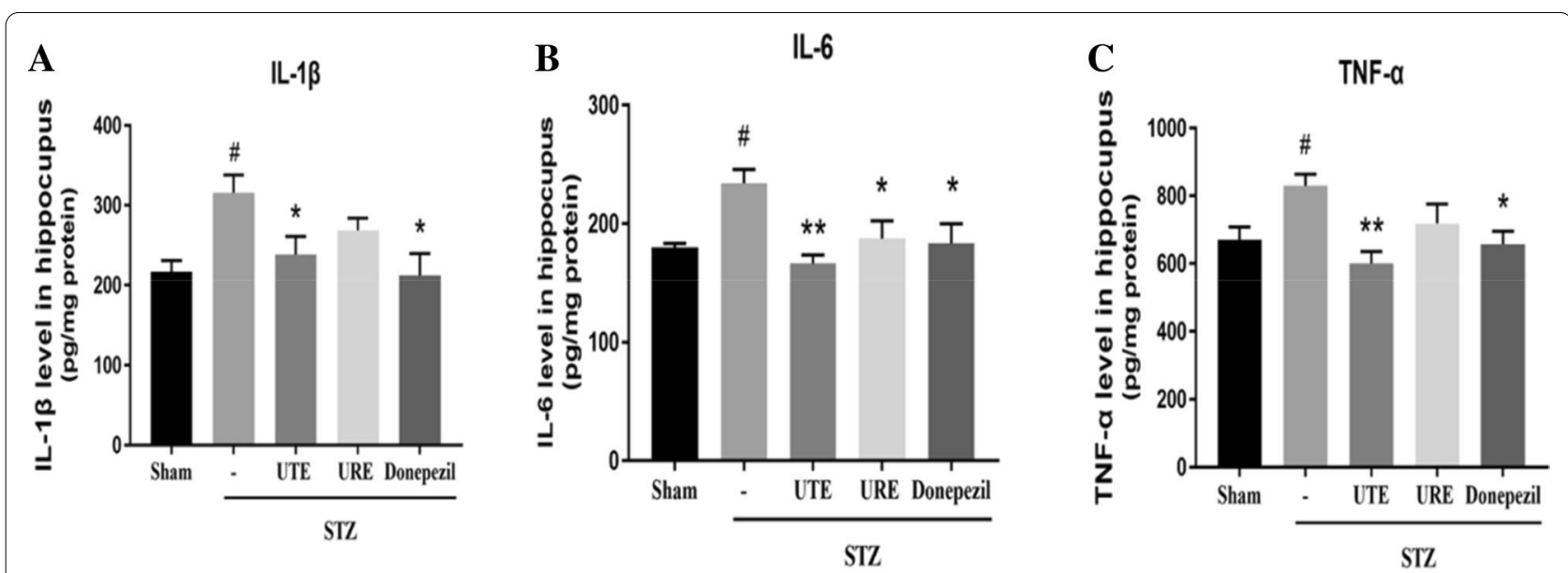

Fig. 4 Effects of UTE and URE on pro-inflammatory cytokines in the STZ-induced AD rat model $(n=5)$. Data were expressed as the mean $\pm S E M$. ${ }^{\#} p<0.05$ and ${ }^{\# \#} p<0.01$ compared with the sham control group. ${ }^{*} p<0.05$ and ${ }^{* *} p<0.01$ compared with the STZ control group 


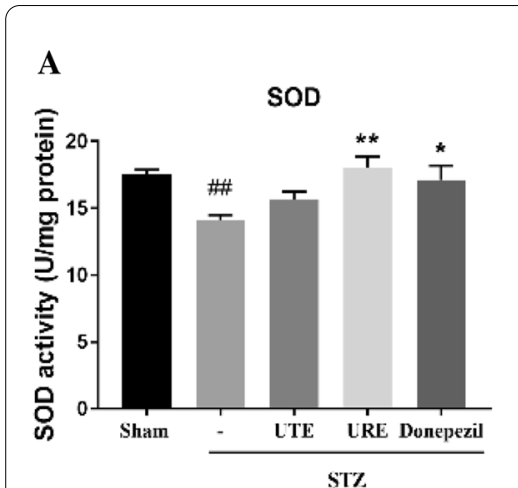

D

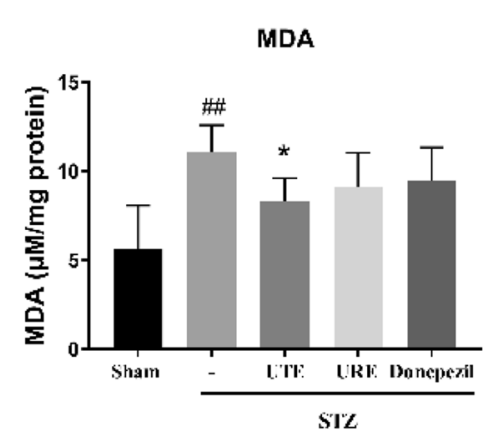

B

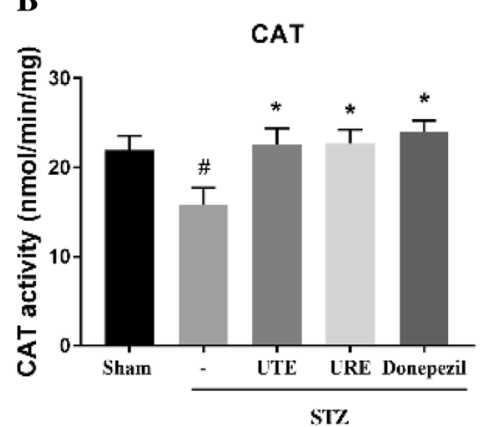

$\mathbf{E}$

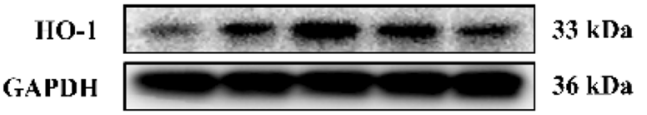

C

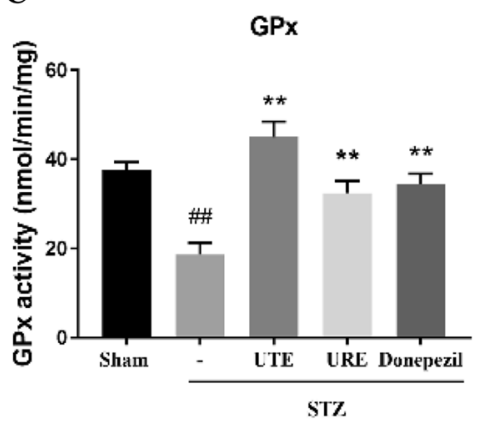

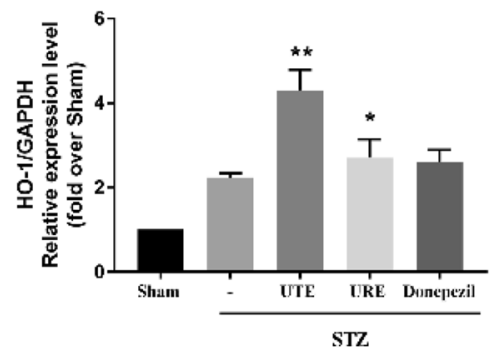

Fig. 5 Effects of UTE and URE on oxidative stress in the STZ-induced AD rat model $(n=5)$. A SOD activity; B CAT activity; C GPx activity; D MDA level; $\mathbf{E}$ The protein expression of HO-1. Data were expressed as the mean \pm SEM. ${ }^{\#} p<0.05$ and ${ }^{\# \#} p<0.01$ compared with the sham control group. ${ }^{*} p<0.05$ and ${ }^{* *} p<0.01$ compared with the STZ control group

The STZ+ vehicle group had markedly lower CAT activity than the Sham group $(p<0.05)$. Compared with the STZ control group, all the treatment groups, including the STZ+ UTE group, the STZ + URE group and the STZ + Donepezil group, significantly increased the levels of CAT activity $(p<0.05)$ (Fig. 5B).

The GPx activity was significantly reduced in the STZ control group $(p<0.01)$ as compared with the Sham group. After treatment, all the treatment groups, including the STZ + UTE group, the STZ + URE group and the STZ + Donepezil group, significantly increased the GPx activity as compared with the STZ control group $(p<0.01)$ (Fig. $5 \mathrm{C}$ ).

When compared with the Sham group, the level of MDA, an oxidative damage marker, markedly accentuated in the STZ control group $(p<0.01)$. UTE treatment markedly reduced the MDA level as compared with the STZ control group $(p<0.05)$. In addition, both the STZ + URE group and the STZ + Donepezil group had lower MDA level than the STZ control group, but the differences were not significantly different (Fig. 5D).

\section{Effects of UTE and URE on the expression of HO-1}

\section{in the STZ-induced AD rats}

The antioxidant effects of UTE and URE were further determined by analyzing the protein expression of $\mathrm{HO}-1$ in the STZ-induced AD rats. There was no difference in the expression of HO-1 between the Sham group and the STZ control group. UTE and URE treatment significantly upregulated the HO-1 protein expression in the brains of the STZ-treated rats $(p<0.01$ and $p<0.05$, respectively) (Fig. 5E).

\section{Effects of UTE and URE on the protein expressions of Keap1} and nuclear Nrf2 in the STZ-induced AD rats

The effects of UTE and URE on the nuclear translocation of Nrf2 were assessed by analyzing the protein expression of Nrf2 and its upstream target Keap1 in the STZinduced AD rats. The western blot results showed that compared with the Sham group, there was a reduction in the protein expression of nuclear Nrf2 in the STZ control group $(p<0.05)$, and the reduction was reversed by 
UTE and URE treatment ( $p<0.05$ for both). In addition, both UTE and URE decreased cytoplasmic Nrf2 protein expression when compared with the Sham group, but the difference was not significant ( $p>0.05$ for both). STZ injection resulted in an increase in the protein expression of Keap1 when compared with the Sham group $(p<0.05)$, which was reduced upon UTE and URE treatment $(p<0.05$ for both) (Fig. 6).

\section{Effects of UTE and URE on Akt/GSK-3 $\beta$ pathway in the STZ-induced AD rats}

The western blot results showed that there were marked reductions in the relative ratios of $\mathrm{p}$-Akt/AKT and $\mathrm{p}$-GSK-3 $\beta /$ GSK- $3 \beta$ in the STZ control group $(p<0.01$ for both), as compared with the Sham group. Both UTE and URE treatment effectively increased the relative ratio of p-Akt/AKT when compared with the STZ control group $(p<0.05$ for both). Similarly, both UTE and URE treatment effectively increased the relative ratio of $p-G S K-3 \beta$ / GSK-3 $\beta$ when compared with STZ control group $(p<0.05$ for both) (Fig. 7).

\section{Discussion}

The present study demonstrated that both UTE and URE could significantly improve the cognitive and memory deficits via alleviating neuroinflammation, rescuing oxidative damage and inhibiting tau hyperphosphorylation through modulating Akt/GSK-3 $\beta$ pathway and Nrf2 activation-translocation.

The genus of Uncaria, belonging to the Rubiaceae family, consists of about 40 species all over the world, including $U$. rhynchophylla and $U$. tomentosa. More than 200 compounds have been isolated from the Uncaria plants up to now, of which the alkaloids exhibit marked pharmacological activities and have been regarded as the active
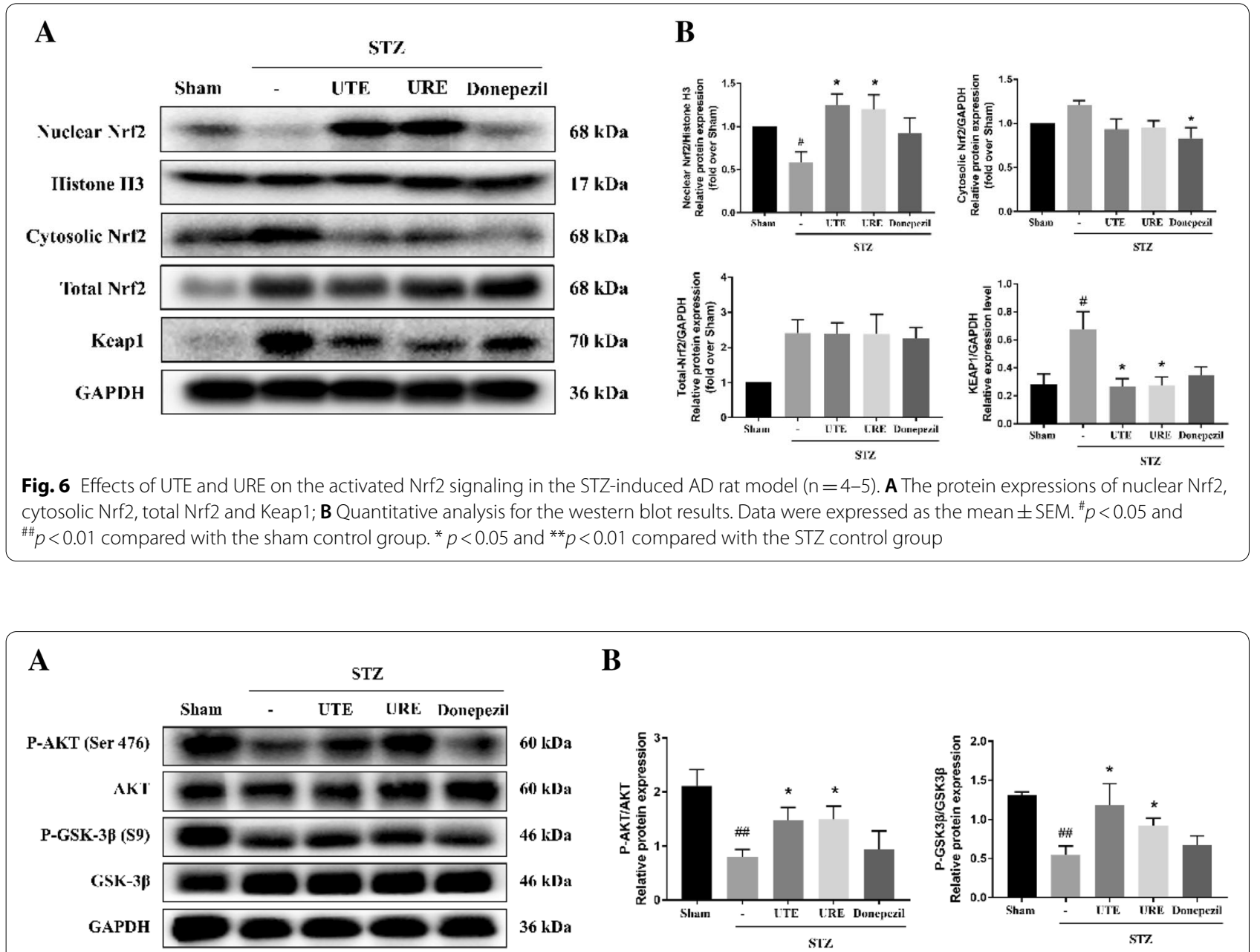

B

Fig. 7 Effects of UTE and URE on Akt/GSK3 $\beta$ pathway in the STZ-induced AD rat model $(n=4-5)$. A The protein expressions of $p$-Akt (S473), Akt, p-GSK-3 $\beta$ (S9), and GSK-3 $\beta$; B Quantitative analysis for the western blot results. Data were expressed as the mean \pm SEM. ${ }^{\#} p<0.05$ and ${ }^{\# \#} p<0.01$ compared with the sham control group. ${ }^{*} p<0.05$ and ${ }^{* *} p<0.01$ compared with the STZ control group 
chemical components for the treatment of AD [32-34]. Among these alkaloids, rhynchophylline (RN), isorhynchophylline (IRN), corynoxeine (CX), and isocorynoxeine (ICX) have been extensively studied, while other alkaloids derived from these Uncaria species have not been systematically studied yet [27, 35]. It was reported that RN, IRN, CX and ICX have the potent inhibitory activity against NO release from lipopolysaccharide (LPS)-activated microglia [36]. Our previous study has demonstrated that IRN $(20 \mathrm{mg} / \mathrm{kg}$ or $40 \mathrm{mg} / \mathrm{kg}$ ) exerted neuroprotective effects and ameliorated cognitive impairments in several animal models of $A D$, including $A \beta_{25-35^{-}}$ induced rats, D-galactose-induced mice and transgenic APP mice [20-22], indicating that IRN is a therapeutic candidate in the treatment of $\mathrm{AD}$. Moreover, our in vitro experiments showed that both IRN and RN markedly decreased the $A \beta$-induced neurotoxicity and apoptosis in PC12 neuronal cells $[18,19,37]$. RN was also found to improve the $A \beta_{1-42}$-induced spatial cognitive impairments in rats [38] and suppress the $A \beta$-induced enhancement of spontaneous discharges in the hippocampal CA1 region of rats [39], suggesting that RN has neuroprotective effects on AD. It has been reported that 5 alkaloids in U. rhynchophylla including IRN and ICX are likely to be vital components for $\mathrm{AD}$ treatment in a potential alkaloid target-AD target network using a network pharmacology approach [33]. RN, IRN CX, and ICX are representative indole alkaloids from $U$. rhynchophylla and $U$. tomentosa. Our HPLC analysis results showed that URE contained $0.478 \%$ RN, $0.163 \%$ IRN, $0.178 \%$ CX, and $0.418 \%$ ICX, and the results were generally congruent with our previous study [17]. UTE was found to be enriched with RN and IRN (0.278\% and $0.531 \%$, respectively) but have much less CX and ICX $(0.010 \%$ and $0.028 \%$, respectively) than URE. Although the contents of UTE and URE varied, both UTE and URE could effectively ameliorate the cognitive and memory deficits in the STZ-induced rats, demonstrating that the protective effects of UTE and URE may be attributed to the synergistic effects of their predominant active component alkaloids such as $\mathrm{RN}$ and IRN.

An ideal animal model of $\mathrm{AD}$ is indispensable to evaluate the potential efficacy of a therapy, and to explore its underlying anti-AD molecular mechanisms. The inappropriate choice of preclinical AD animal models might result in the high failure rate of drug development in clinical trials [40]. As we know, $\mathrm{AD}$ can be divided into familial AD (fAD) and sporadic AD (sAD). Although fAD is much less common (accounting for only $5 \%$ of all AD cases), many anti-AD drugs under clinical development were evaluated based on the fAD animal models, in which the underlying mechanisms are primarily associated with familial mutations [40, 41]. Patients diagnosed with sAD make up over $95 \%$ of all cases and have no documented familial history of AD [42, 43]. The present study adopted ICV administration of STZ in rats to establish sporadic AD model. STZ is a natural alkylating anti-neoplastic agent which is selectively toxic for insulin producing cells both in the periphery and in the brain. It has been well recognized as an appropriate sporadic $\mathrm{AD}$ model closely resembling $\mathrm{AD}$ in humans in terms of progressive memory deficits, abnormal cerebral glucose and energy metabolism, decreased expressions of insulin receptor (IR), induction of hyperphosphorylated tau as well as $A \beta$ plaque deposition [44, 45]. In addition, Mehla et al. [46] found that the cognitive deficits induced by both single and twice ICV-STZ injections ( $3 \mathrm{mg} / \mathrm{kg}$ ) persist from the 2nd week after STZ injection to the end of the 14-week experiment. In the present study, the MWM results showed that a significant decrease was found in the number of target quadrant crossing and time spent in the target quadrant in the STZ treated group in comparison with the sham group, indicating that STZ could cause cognitive impairments and the STZ-induced AD model was successfully established. In addition, we also found that STZ could induce many pathological features of $\mathrm{AD}$, including neuroinflammation, oxidative stress and hyperphosphorylation of tau protein. Both UTE and URE treatment could ameliorate the cognitive deficits induced by STZ in rats.

Neuroinflammation has been well recognized as a prominent contributor in $\mathrm{AD}$, involving in not only the onset, but also the pathological process of $\mathrm{AD}[47$, 48]. The release of pro-inflammatory markers, such as IL- $1 \beta$, IL- 6 and TNF- $\alpha$, can cause neuronal damage, synaptic dysfunction and inhibition of neurogenesis [49]. IL-1 is a major driver of inflammation which has a clear detrimental effect and causes neuronal injury in $\mathrm{AD}$ and other chronic neurodegenerative disorders [50, $51]$. It has been indicated that IL-1 $\beta$ is closely associated with neurodegeneration and impaired learning and memory functions [52]. IL- $1 \beta$ is elevated in AD patients, along with its antagonist IL-1Ra and its soluble receptor sIL-1R1 [50]. Similarly, as the main proinflammatory cytokines in the brain, IL- 6 and TNF- $\alpha$ have been extensively investigated for their roles in $\mathrm{AD}$ pathology. Studies showed that the levels of IL-6 and TNF- $\alpha$ were elevated in the brain and in cerebrospinal fluid (CSF) of AD patients, while IL- 6 may be a predictor for the severity of cognitive defects $[53,54]$. In line with these findings, the increased levels of these proinflammatory cytokines were also observed in the AD animal models [55]. Moreover, neuroinflammation and microglial activation can exacerbate tau pathology by promoting hyperphosphorylation and aggregation of tau protein [56]. Thus, anti-inflammatory intervention 
has been identified as a potential therapeutic approach for this disease [57]. The present study revealed that both UTE and URE exerted anti-neuroinflammatory action via reducing the elevated levels of IL-1 $\beta$, IL-6 and TNF- $\alpha$ in the STZ-induced AD rat model.

Oxidative stress, characterized by an imbalance in reactive oxygen species (ROS) and anti-oxidative defense, is an early feature of $\mathrm{AD}$ and plays a critical role in $\mathrm{AD}$ pathogenesis [58]. The impairment of anti-oxidative systems in the AD brain leads to the decreased activities of antioxidant enzymes (e.g. SOD, CAT and GPx) and the increased generation of free radicals, thereby causing neuronal damage by promoting lipid peroxidation, protein breakdown and DNA damage [59]. Lipid peroxidation, mediated by superoxide, can directly contribute to the blood brain barrier (BBB) disruption, leading to the influx into the brain of inflammatory mediators and neurotoxic blood-derived debris, and these events subsequently initiate multiple mechanisms of neurodegeneration of $\mathrm{AD}$ [60]. In addition, the end products of lipid peroxidation, such as MDA and 4-hydroxy2-nonenal (4-HNE), are found to be increased in the AD brain [61]. Emerging evidence suggested that interventions targeting anti-oxidative bioavailability are potential therapeutic strategies for AD [62]. The present study demonstrated that both UTE and URE exerted anti-oxidative effects by enhancing the activities of antioxidant enzymes (SOD, CAT and GPx) and increasing the protein expression of HO-1. Moreover, UTE could decrease the MDA level.

Tau protein is of great importance for stabilizing microtubules under physiological conditions [63]. In the context of $\mathrm{AD}$, the pathological modification of tau, i.e., hyperphosphorylated tau, detaches from microtubules, aggregates into paired helical filaments (PHFs) and forms neurotoxic NFTs, causing synaptic loss, neuronal dysfunction, and eventually cognitive impairment [64]. Thus, the progression of AD inevitably accompanies with abnormal hyperphosphorylation and aggregation of tau protein. Furthermore, hyperphosphorylated tau pathology correlates with symptom severity, and is more closely related to cognitive dysfunction than amyloid deposition in $\mathrm{AD}$ patients [65]. Overwhelming evidence demonstrated that there are prominently increased levels of phosphorylated tau (p-tau) at the sites of Thr181, Ser396 and Ser404 in AD patients [66]. Recent study revealed that $\mathrm{p}$-tau Thr181 is a highly novel biomarker for AD diagnosis [67], while p-tau Ser396 and p-tau Ser404 are also found to play an important role in AD pathology [68]. The present study showed that UTE could reverse the elevated expressions of total tau, p-tau Ser396 and p-tau Ser404 while URE could reverse the elevated expressions of total tau, p-tau Ser181 and p-tau Ser396, indicating that suppressive effects of UTE and URE on these specific hyperphosphorylation sites of tau protein is one of the mechanisms underlying their cognitive improving properties.

$\mathrm{Nrf2}$, the master regulator of homeostatic responses, is considered as a promising therapeutic target for $\mathrm{AD}$ and an essential component in modulation of oxidative stress and neuroinflammation in AD [69]. The Nrf2 transcriptional activity declines with age [70], and it was reported that not only nuclear Nrf2 levels, but also Nrf2-mediated transcription are suppressed in the hippocampus of AD patients [71]. Nrf2 deficiency worsens APP and Tau pathology and exacerbates cognitive deficits in preclinical AD models, which is associated with increased levels of oxidative and pro-inflammatory markers [72]. Conversely, up-regulation of Nrf2 by intra-hippocampal injection can restore spatial learning impairment in transgenic $\mathrm{AD}$ mice [73]. In an inactive state, the transcriptional role of $\mathrm{Nrf2}$ is repressed, due to its binding to Kelch-like ECH associated protein 1 (Keap 1 ) in the cytoplasm [74]. Many studies demonstrated that inactive GSK-3 $\beta$ activity can alter the conformation of Keap1-Nrf2 complex and disrupt their interaction, thereby increasing Nrf2 nuclear translocation and Nrf2 related downstream antioxidant gene expression (such as HO-1, NQO- 1 and CAT) $[75,76]$. Unlike most cellular kinases that are normally active, GSK-3 $\beta$ is inactive under phosphorylation (Ser9) that is regulated by activated Akt. GSK-3 $\beta$ activation is elevated [77] and regulates tau phosphorylation [78] in AD patients. Conditional overexpression of GSK-3 $\beta$ causes neuronal death, hyperphosphorylation of tau protein and cognitive impairments in mice [79], while GSK-3 $\beta$ inhibitors have been found to reverse cognitive deficits in several rodent models of $\mathrm{AD}$ [80]. It has been demonstrated that the PI3K/Akt/ GSK-3 $\beta$ pathway plays a pivotal role in the regulation of Nrf2 nuclear export and degradation in AD [81]. Recent studies targeted Akt/GSK-3 $\beta$-mediated Nrf2 activation as a therapeutic potential in CNS diseases, such as AD [82] and ischemic stroke [83]. This study showed that increased protein expression of nuclear Nrf2 is accompanied with an increase of Akt activation (phosphorylation) and GSK3 $\beta$ inactivation (phosphorylation) after UTE and URE treatment in the STZ-induced AD model, indicating that Akt(Ser473)/GSK3 $\beta$ (Ser9) may mediate the neuroprotective effects of UTE and URE.

\section{Conclusion}

In this present study, we indicated for the first time that UTE and URE, the two herbal products with similar chemical constituents, could alleviate cognitive impairments via suppressing tau hyperphosphorylation in the STZ-induced AD rats, and the effects are largely attributed to their anti-oxidant and anti-neuroinflammatory 


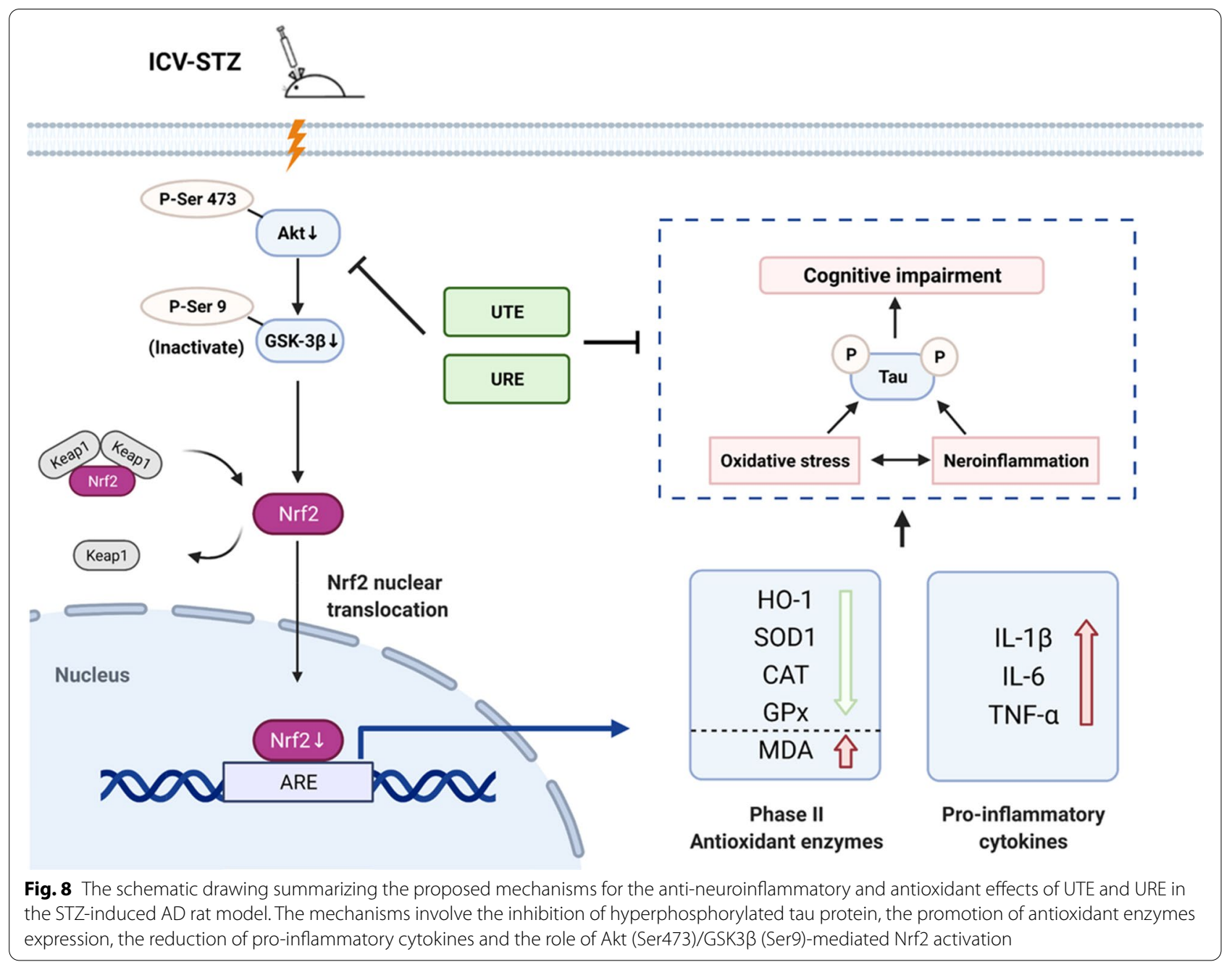

activities via Akt (Ser473)/GSK3 $\beta$ (Ser9)-mediated Nrf2 activation (Fig. 8). Furthermore, this study provides preclinical evidence for the comparative effects of UTE and URE on AD, suggesting that both UTE and URE can be potential candidates for further clinical trials. Finally, the major bioactive components in UTE and URE are worthy of further investigation for the anti-AD effects.

\section{Abbreviations}

4-HNE: 4-Hydroxy2-nonenal; Aß: Beta-amyloid; AChEls: Acetylcholinesterase inhibitors; AD: Alzheimer's disease; BBB: Blood brain barrier; CAT: Catalase; CSF: Cerebrospinal fluid; ECL: Enhanced chemiluminescence; GPx: Glutathione peroxidase; HPLC: High-performance liquid chromatography; ICV: Intracerebroventricular; IL-1 $\beta$ : Interleukin-1 $\beta$; IRN: Isorhynchophylline; Keap 1: Kelchlike ECH associated protein 1; MDA: Malondialdehyde; MWM: Morris water maze; NFTs: Neurofibrillary tangles; NMDA: N-Methyl-D-aspartate; PHFs: Paired helical filaments; PVDF: Polyvinyl difluoride; ROS: Reactive oxygen species; SD: Sprague-Dawley; SOD: Superoxide dismutases; STZ: Streptozotocin; TNF-a: Tumor necrosis factor alpha; UTE: Extract of Uncaria tomentosa; URE: Extract of Uncaria rhynchophylla.

\section{Acknowledgements}

Not applicable.

\section{Authors' contributions}

YFX, ZXL and PCS conceived the research idea and designed the experimental protocols. QQX performed the animal studies and collected the experimental data. ZH and SPI constructed the HPLC profiles. QQX and WY performed the data analysis. QQX drafted the manuscript. PCS, YFX and ZXL revised the manuscript. All authors read and approved the final manuscript.

\section{Funding}

This work was supported by a grant from Li Dak Sum Yip Yio Chin R \& D Centre for Chinese Medicine, The Chinese University of Hong Kong.

\section{Availability of data and materials}

I agree to share my data and materials.

\section{Declarations}

\section{Ethics approval and consent to participate}

Approval for the experimental protocol was obtained from the Animal Experimentation Ethics Committee of The Chinese University of Hong Kong (Ref. No.18/264/MIS). 


\section{Consent for publication \\ Not applicable.}

\section{Competing interests}

The authors declare that there is no conflict of interests regarding the publication of this paper.

\section{Author details}

${ }^{1}$ School of Chinese Medicine, Faculty of Medicine, The Chinese University of Hong Kong, Shatin, N.T., Hong Kong SAR, People's Republic of China. ${ }^{2}$ School of Life Sciences, Faculty of Science, The Chinese University of Hong Kong, Shatin, N.T., Hong Kong SAR, People's Republic of China. ${ }^{3}$ Li Dak Sum Yip Yio Chin R\&D Centre for Chinese Medicine, The Chinese University of Hong Kong, Shatin, N.T., Hong Kong SAR, People's Republic of China. ${ }^{4}$ Hong Kong Institute of Integrative Medicine, The Chinese University of Hong Kong, Hong Kong, Shatin, N.T., Hong Kong SAR, People's Republic of China.

Received: 15 July 2021 Accepted: 6 October 2021

Published online: 27 October 2021

\section{References}

1. Alzheimer's Association. Alzheimer's disease facts and figures. Alzheimers Dement. 2021;17(3):327-406.

2. Jia L, Du Y, Chu L, Zhang Z, Li F, Lyu D, et al. Prevalence, risk factors, and management of dementia and mild cognitive impairment in adults aged 60 years or older in China: a cross-sectional study. Lancet Public Health. 2020;5:e661-71.

3. World Health Organization. The top 10 causes of death; 2020. https:// www.who.int/news-room/fact-sheets/detail/the-top-10-causes-of-death. Accessed 20 Mar 2021.

4. James BD, Bennett DA. Causes and patterns of dementia: an update in the era of redefining Alzheimer's disease. Annu Rev Public Health. 2019:2019(40):65-84.

5. Querfurth HW, LaFerla FM. Alzheimer's disease. N Engl J Med. 2010;362(4):329-44

6. Chaves S, Várnagy K, Santos MA. Recent multi-target approaches on the development of anti-Alzheimer's agents integrating metal chelation activity. Curr Med Chem. 2021. https://doi.org/10.2174/092986732866621 0218183032.(Inpress).

7. Morris GP, Clark IA, Vissel B. Questions concerning the role of amyloid- $\beta$ in the definition, aetiology and diagnosis of Alzheimer's disease. Acta neuropathol. 2018;136:663-89.

8. Schmidt R, Hofer E, Bouwman FH, Buerger K, Cordonnier C, Fladby T, et al. EFNS-ENS/EAN Guideline on concomitant use of cholinesterase inhibitors and memantine in moderate to severe Alzheimer's disease. Eur J neurol. 2015;22:889-98.

9. Huang LK, Chao SP, Hu CJ. Clinical trials of new drugs for Alzheimer disease. J Biomed Sci. 2020;27:18.

10. Arvanitakis Z, Shah RC, Bennett DA. Diagnosis and management of dementia: review. JAMA. 2019;322:1589-99.

11. Pan RY, Ma J, Kong XX, Wang XF, Li SS, Qi XL, et al. Sodium rutin ameliorates Alzheimer's disease-like pathology by enhancing microglial amyloid- $\beta$ clearance. Sci Adv. 2019;5:eaau6328.

12. Noori T, Dehpour AR, Sureda A, Sobarzo-Sanchez E, Shirooie S. Role of natural products for the treatment of Alzheimer's disease. Eur J Pharmacol. 2021;898:173974.

13. Yang WT, Zheng XW, Chen S, Shan CS, Xu QQ, Zhu JZ, et al. Chinese herbal medicine for Alzheimer's disease: Clinical evidence and possible mechanism of neurogenesis. Biochem Pharmacol. 2017;141:143-55.

14. Yang W, Liu Y, Xu QQ, Xian YF, Lin ZX. Sulforaphene ameliorates neuroinflammation and hyperphosphorylated tau protein via regulating the PI3K/Akt/GSK-3ß pathway in experimental models of Alzheimer's disease. Oxid Med Cell Longev. 2020;2020:4754195

15. Watanabe H, Zhao Q, Matsumoto K, Tohda M, Murakami Y, Zhang SH, et al. Pharmacological evidence for antidementia effect of Choto-san (Gouteng-san), a traditional Kampo medicine. Pharmacol Biochem Behav. 2003;75:635-43.

16. Tabuchi M, Yamaguchi T, lizuka S, Imamura S, Ikarashi Y, Kase Y. Ameliorative effects of yokukansan, a traditional Japanese medicine, on learning and non-cognitive disturbances in the Tg2576 mouse model of Alzheimer's disease. J Ethnopharmacol. 2009;122:157-62.

17. Xian YF, Lin ZX, Zhao M, Mao QQ, Ip SP, Che CT. Uncaria rhynchophylla ameliorates cognitive deficits induced by D-galactose in mice. Planta Med. 2011;77:1977-83.

18. Xian YF, Lin ZX, Mao QQ, Ip SP, Su ZR, Lai XP. Protective effect of isorhynchophylline against $\beta$-amyloid-induced neurotoxicity in PC12 cells. Cell Mol Neurobiol. 2012;32:353-60.

19. Xian YF, Lin ZX, Mao QQ, Chen JN, Su ZR, Lai XP, et al. Isorhynchophylline protects PC12 cells against beta-amyloid-induced apoptosis via PI3K/Akt signaling pathway. Evid Based Complement Alternat Med. 2013;2013:163057.

20. Xian YF, Mao QQ, Wu JC, Su ZR, Chen JN, Lai XP, et al. Isorhynchophylline treatment improves the amyloid- $\beta$-induced cognitive impairment in rats via inhibition of neuronal apoptosis and tau protein hyperphosphorylation. J Alzheimers Dis. 2014;39(2):331-46.

21. Xian YF, Su ZR, Chen JN, Lai XP, Mao QQ, Cheng CH, et al. Isorhynchophylline improves learning and memory impairments induced by D-galactose in mice. Neurochem Int. 2014;76:42-9.

22. Li HQ, Ip SP, Yuan QJ, Zheng GQ, Tsim K, Dong T, et al. Isorhynchophylline ameliorates cognitive impairment via modulating amyloid pathology, tau hyperphosphorylation and neuroinflammation: Studies in a transgenic mouse model of Alzheimer's disease. Brain Behav Immun. 2019;82:264-78.

23. Erowele Gl, Kalejaiye AO. Pharmacology and therapeutic uses of cat's claw. Am J Health Syst Pharm. 2009;66:992-5.

24. Sandoval M, Charbonnet RM, Okuhama NN, Roberts J, Krenova Z, Trentacosti AM, et al. Cat's claw inhibits TNFalpha production and scavenges free radicals: role in cytoprotection. Free Radic Biol Med. 2000;29:71-8.

25. Hardin SR. Cat's claw: an Amazonian vine decreases inflammation in osteoarthritis. Complement Ther Clin Pract. 2007:13:25-8.

26. Keplinger K, Laus G, Wurm M, Dierich MP, Teppner H. Uncaria tomentosa (Willd.) DC.-ethnomedicinal use and new pharmacological, toxicological and botanical results. J Ethnopharmacol. 1999;64:23-34.

27. Zhang Q, Zhao JJ, Xu J, Feng F, Qu W. Medicinal uses, phytochemistry and pharmacology of the genus Uncaria. J Ethnopharmacol. 2015;173:48-80.

28. Snow AD, Castillo GM, Nguyen BP, Choi PY, Cummings JA, Cam J, et al. The Amazon rain forest plant Uncaria tomentosa (cat's claw) and its specific proanthocyanidin constituents are potent inhibitors and reducers of both brain plaques and tangles. Sci Rep. 2019:9:561.

29. Castilhos LG, Oliveira JS, Adefegha SA, Manzoni AG, Passos DF, Assmann $\mathrm{CE}$, et al. Uncaria tomentosa improves cognition, memory and learning in middle-aged rats. Exp Gerontol. 2020;138:111016.

30. $\mathrm{ICH}$. Topic Q2 (R1): validation of analytical procedures: text and methodology. In: International conference on harmonization (ICH), IFPMA, Geneva, Switzerland; 2005.

31. Paxinos $G$, Watson $C$. The rat brain in stereotaxic coordinates. Amsterdam: Elsevier; 2013.

32. Shi JS, Yu JX, Chen XP, Xu RX. Pharmacological actions of Uncaria alkaloids, rhynchophylline and isorhynchophylline. Acta Pharmacol Sin. 2003:24:97-101.

33. Zeng P, Wang XM, Ye CY, Su HF, Tian Q. The main alkaloids in Uncaria rhynchophylla and their anti-Alzheimer's disease mechanism determined by a network pharmacology approach. Int J Mol Sci. 2021;22:3612.

34. Yang W, Ip SP, Liu L, Xian YF, Lin ZX. Uncaria rhynchophylla and its major constituents on central nervous system: a review on their pharmacological actions. Curr Vasc Pharmacol. 2020;18:346-57.

35. Wei S, Luo Z, Cui S, Qiao J, Zhang Z, Zhang L, et al. Molecular identification and targeted quantitative analysis of medicinal materials from Uncaria species by DNA barcoding and LC-MS/MS. Molecules. 2019;24:175.

36. Yuan D, Ma B, Wu C, Yang J, Zhang L, Liu S, et al. Alkaloids from the leaves of Uncaria rhynchophylla and their inhibitory activity on NO production in lipopolysaccharide-activated microglia. J Nat Prod. 2008;71:1271-4.

37. Xian YF, Lin ZX, Mao QQ, Hu Z, Zhao M, Che CT, et al. Bioassay-guided isolation of neuroprotective compounds from Uncaria rhynchophylla against beta-amyloid-induced neurotoxicity. Evid Based Complement Alternat Med. 2012;2012:802625

38. Yang Y, Ji WG, Zhu ZR, Wu YL, Zhang ZY, Qu SC. Rhynchophylline suppresses soluble $A \beta 1-42$-induced impairment of spatial cognition function 
via inhibiting excessive activation of extrasynaptic NR2B-containing NMDA receptors. Neuropharmacology. 2018;135:100-12.

39. Shao H, Mi Z, JiWG, Zhang CH, Zhang T, Ren SC, Zhu ZR. Rhynchophylline protects against the amyloid $\beta$-induced increase of spontaneous discharges in the hippocampal CA1 region of rats. Neurochem Res. 2015;40:2365-73.

40. Zhang L, Chen C, Mak MS, Lu J, Wu Z, Chen Q, et al. Advance of sporadic Alzheimer's disease animal models. Med Res Rev. 2020;40(1):431-58.

41. Mangialasche F, Solomon A, Winblad B, Mecocci P, Kivipelto M. Alzheimer's disease: clinical trials and drug development. Lancet Neurol. 2010;9(7):702-16.

42. Mattsson N, Zetterberg H, Hansson O, Andreasen N, Parnetti L, Jonsson M, et al. CSF biomarkers and incipient Alzheimer disease in patients with mild cognitive impairment. JAMA. 2009;302(4):385-93.

43. Bali J, Gheinani AH, Zurbriggen S, Rajendran L. Role of genes linked to sporadic Alzheimer's disease risk in the production of $\beta$-amyloid peptides. Proc Natl Acad Sci U S A. 2012;109(38):15307-11.

44. Ravelli KG, Rosário BD, Camarini R, Hernandes MS, Britto LR. Intracerebroventricular streptozotocin as a model of Alzheimer's disease: neurochemical and behavioral characterization in mice. Neurotox Res. 2017;31(3):327-33.

45. Halawany A, Sayed N, Abdallah HM, Dine R. Protective effects of gingerol on streptozotocin-induced sporadic Alzheimer's disease: emphasis on inhibition of $\beta$-amyloid, COX-2, alpha-, beta - secretases and APH1a. Sci Rep. 2017;7(1):2902.

46. Mehla J, Pahuja M, Gupta YK. Streptozotocin-induced sporadic Alzheimer's disease: selection of appropriate dose. J Alzheimers Dis. 2013;33(1):17-21.

47. Calsolaro V, Edison P. Neuroinflammation in Alzheimer's disease: current evidence and future directions. Alzheimers Dement. 2016;12(6):719-32.

48. Pereira CF, Santos AE, Moreira PI, Pereira AC, Sousa FJ, Cardoso SM, et al. Is Alzheimer's disease an inflammasomopathy? Ageing Res Rev. 2019;56:100966.

49. Lyman M, Lloyd DG, Ji X, Vizcaychipi MP, Ma D. Neuroinflammation: the role and consequences. Neurosci Res. 2014;79:1-12.

50. Italiani P, Puxeddu I, Napoletano S, Scala E, Melillo D, Manocchio S, et al. Circulating levels of IL-1 family cytokines and receptors in Alzheimer's disease: new markers of disease progression? J Neuroinflammation. 2018;15(1):342.

51. Allan SM, Tyrrell PJ, Rothwell NJ. Interleukin-1 and neuronal injury. Nat Rev Immunol. 2005;5(8):629-40.

52. Shaftel SS, Griffin WS, O'Banion MK. The role of interleukin-1 in neuroinflammation and Alzheimer disease: an evolving perspective. J Neuroinflammation. 2008;5:7.

53. Lai K, Liu CS, Rau A, Lanctôt KL, Köhler CA, Pakosh M, et al. Peripheral inflammatory markers in Alzheimer's disease: a systematic review and metaanalysis of 175 studies. J Neurol Neurosurg Psychiatry. 2017;88(10):876-82.

54. Brosseron F, Krauthausen M, Kummer M, Heneka MT. Body fluid cytokine levels in mild cognitive impairment and Alzheimer's disease: a comparative overview. Mol Neurobiol. 2014;50(2):534-44.

55. Babcock AA, Ilkjær L, Clausen BH, Villadsen B, Dissing-Olesen L, Bendixen AT, et al. Cytokine-producing microglia have an altered beta-amyloid load in aged APP/PS1 Tg mice. Brain Behav Immun. 2015;48:86-101.

56. Leng F, Edison P. Neuroinflammation and microglial activation in Alzheimer disease: where do we go from here? Nat Rev Neurol. 2021;17(3):157-72.

57. Scheltens $\mathrm{P}$, De Strooper B, Kivipelto M, Holstege H, Chételat G, Teunissen CE, et al. Alzheimer's disease. Lancet. 2021;397(10284):1577-90.

58. Butterfield DA, Halliwell B. Oxidative stress, dysfunctional glucose metabolism and Alzheimer disease. Nat Rev Neurosci. 2019;20(3):148-60.

59. Wojsiat J, Zoltowska KM, Laskowska-Kaszub K, Wojda U. Oxidant/antioxidant imbalance in Alzheimer's disease: therapeutic and diagnostic prospects. Oxid Med Cell Longev. 2018;2018:6435861.

60. Butterfield DA. Brain lipid peroxidation and alzheimer disease: Synergy between the Butterfield and Mattson laboratories. Ageing Res Rev. 2020;64:101049

61. Sweeney MD, Sagare AP, Zlokovic BV. Blood-brain barrier breakdown in Alzheimer disease and other neurodegenerative disorders. Nat Rev Neurol. 2018;14(3):133-50

62. Simunkova M, Alwasel SH, Alhazza IM, Jomova K, KollarV, Rusko M, et al. Management of oxidative stress and other pathologies in Alzheimer's disease. Arch Toxicol. 2019;93(9):2491-513.

63. Lasagna-Reeves CA, Castillo-Carranza DL, Sengupta U, Sarmiento J, Troncoso J, Jackson GR, et al. Identification of oligomers at early stages of tau aggregation in Alzheimer's disease. FASEB J. 2012;26(5):1946-59.
64. Gao YL, Wang N, Sun FR, Cao XP, Zhang W, Yu JT. Tau in neurodegenerative disease. Ann Transl Med. 2018;6(10):175.

65. Dujardin S, Commins C, Lathuiliere A, Beerepoot P, Fernandes AR, Kamath $T V$, et al. Tau molecular diversity contributes to clinical heterogeneity in Alzheimer's disease. Nat Med. 2020;26(8):1256-63.

66. Brickman AM, Manly JJ, Honig LS, Sanchez D, Reyes-Dumeyer D, Lantigua RA, et al. Plasma p-tau181, p-tau217, and other blood-based Alzheimer's disease biomarkers in a multi-ethnic, community study. Alzheimers Dement. 2021:17(8):1353-64.

67. Karikari TK, Pascoal TA, Ashton NJ, Janelidze S, Benedet AL, Rodriguez JL, et al. Blood phosphorylated tau 181 as a biomarker for Alzheimer's disease: a diagnostic performance and prediction modelling study using data from four prospective cohorts. Lancet Neurol. 2020;19(5):422-33.

68. Blennow K, Hampel H. CSF markers for incipient Alzheimer's disease. Lancet Neurol. 2003;2(10):605-13.

69. Osama A, Zhang J, Yao J, Yao X, Fang J. Nrf2: a dark horse in Alzheimer's disease treatment. Ageing Res Rev. 2020;64:101206.

70. Rahman MM, Sykiotis GP, Nishimura M, Bodmer R, Bohmann D. Declining signal dependence of Nrf2-MafS-regulated gene expression correlates with aging phenotypes. Aging Cell. 2013;12(4):554-62.

71. Ramsey CP, Glass CA, Montgomery MB, Lindl KA, Ritson GP, Chia LA, et al. Expression of Nrf2 in neurodegenerative diseases. J Neuropathol Exp Neurol. 2007;66(1):75-85.

72. Ren P, Chen J, Li B, Zhang M, Yang B, Guo X, et al. Nrf2 ablation promotes Alzheimer's disease-like pathology in APP/PS1 transgenic mice: the role of neuroinflammation and oxidative stress. Oxid Med Cell Longev. 2020:2020:3050971.

73. Kanninen $\mathrm{K}$, Heikkinen R, Malm T, Rolova T, Kuhmonen S, Leinonen H, et al. Intrahippocampal injection of a lentiviral vector expressing Nrf2 improves spatial learning in a mouse model of Alzheimer's disease. Proc Natl Acad Sci U S A. 2009:106(38):16505-10.

74. Zhang DD, Hannink M. Distinct cysteine residues in Keap1 are required for Keap 1-dependent ubiquitination of Nrf2 and for stabilization of Nrf2 by chemopreventive agents and oxidative stress. Mol Cell Biol. 2003;23(22):8137-51.

75. Rojo Al, Rada P, Egea J, Rosa AO, López MG, Cuadrado A. Functional interference between glycogen synthase kinase-3 beta and the transcription factor Nrf2 in protection against kainate-induced hippocampal cell death. Mol Cell Neurosci. 2008;39(1):125-32.

76. Rojo Al, Sagarra MR, Cuadrado A. GSK-3beta down-regulates the transcription factor Nrf2 after oxidant damage: relevance to exposure of neuronal cells to oxidative stress. J Neurochem. 2008;105(1):192-202.

77. Leroy K, Yilmaz Z, Brion JP. Increased level of active GSK-3beta in Alzheimer's disease and accumulation in argyrophilic grains and in neurones at different stages of neurofibrillary degeneration. Neuropathol Appl Neurobiol. 2007;33(1):43-55.

78. Pei JJ, Braak E, Braak H, Grundke-lqbal I, lqbal K, Winblad B, et al. Distribution of active glycogen synthase kinase 3beta (GSK-3beta) in brains staged for Alzheimer disease neurofibrillary changes. J Neuropathol Exp Neurol. 1999;58(9):1010-9.

79. Engel T, Hernández F, Avila J, Lucas JJ. Full reversal of Alzheimer's disease-like phenotype in a mouse model with conditional overexpression of glycogen synthase kinase-3. J Neurosci. 2006;26(19):5083-90.

80. King MK, Pardo M, Cheng Y, Downey K, Jope RS, Beurel E. Glycogen synthase kinase-3 inhibitors: Rescuers of cognitive impairments. Pharmacol Ther. 2014;141(1):1-12.

81. Fão L, Mota SI, Rego AC. Shaping the Nrf2-ARE-related pathways in Alzheimer's and Parkinson's diseases. Ageing Res Rev. 2019;54:100942.

82. Zhou Y, Xie N, Li L, Zou Y, Zhang X, Dong M. Puerarin alleviates cognitive impairment and oxidative stress in APP/PS1 transgenic mice. Int J Neuropsychopharmacol. 2014;17(4):635-44.

83. Liao S, Wu J, Liu R, Wang S, Luo J, Yang Y, et al. A novel compound DBZ ameliorates neuroinflammation in LPS-stimulated microglia and ischemic stroke rats: Role of Akt(Ser473)/GSK3ß(Ser9)-mediated Nrf2 activation. Redox Boil. 2020;36:101644

\section{Publisher's Note}

Springer Nature remains neutral with regard to jurisdictional claims in published maps and institutional affiliations. 